\title{
Liaison-psychiatry: reasons for consultation, diagnosis, and differences between medical and surgical patients
}

\author{
S. Ruiz-Doblado ${ }^{1}$, N. Atienza-Martinez ${ }^{2}$, J.R. Lacalle-Remigio ${ }^{2}$ \\ ${ }^{1}$ Consultation-Liaison Psychiatric Unit, Merced County Hospital Osuna (Seville), Spain; ${ }^{2}$ Biostatistics Unit, School of Medicine, University of \\ Seville Seville, Spain
}

There are various aims of consultation-liaison psychiatry in the general hospital: offering quality assistance to medical-surgical patients admitted to hospital, teaching non-psychiatrist professionals, and research into aspects common to both psychiatry and medicine [3]. The most frequent reasons for consultation requests were the physician's difficulty in making a diagnosis, depressive and anxiety illness, suicidal attempts, somatic symptoms with no medical explanation, confusional or agitated states, substance abuse or dependence, problems in the doctor-patient relationship, refusal to carry out complementary tests or treatments, and the existence of psychiatric backgrounds [1]. Discord between the reason for consultation and the psychiatric diagnosis is commonplace, everything is usually considered as depression and organic mental disorders go under-diagnosed $[2,4]$. Indeed, in spite of the fact that the overall rate of medical consultations is usually greater than surgical consultations, little is known about the differences in profiles of patients consulting doctors and of those who consult surgeons.

A simple, random sample $(n=160)$ of medical $(84$ cases) and surgical patients ( 76 cases) was taken from the Liaison Psychiatry Unit of Osuna County Hospital (Seville, Spain). This public hospital covers a rural area with a population of approximately 170000 inhabitants. Sociodemographic and clinical variables were recorded in order to carry out a general descriptive study, including the most frequent reasons for consultation and ICD-10 diagnoses [5], as was diagnostic identifi- cation or concordance between the findings of medical doctors and those of the psychiatrist. Differences in profile between medical and surgical patients were also assessed. Chi-square and ANOVA tests were used, as well as stratified analysis for adjusting differences in gender and age distribution (Mantel-Haenszel test).

The most relevant results from descriptive study and diagnostic identification by non-psychiatrist physicians can be found in table I. The most frequent reasons for consultation and psychiatric diagnoses were similar to those found in other studies [2]. Organic mental disorders (F00-F09, mainly Delirium) predominated in our series. Under-identification of the organic mental disorders group (F00-F09) is commonplace [2], pointing to the difficulty non-psychiatrist physicians have in recognizing underlying organic causes of acute confusional states. Those patients from the ICD-10 anxiousneurotic-somatoform group were the most problematic for doctors when trying to reach a differential diagnosis (20.7\% of the total number of neurotics). There were also problems with the doctor-patient rapport in this group, as well as refusals to carry out diagnostic tests or treatments (13.8\% of the total number of neurotics).

Concerning differences between medical and surgical patients, there were more medical patients with substance-related disorders (F10-F19) and mood disorders (F30-F39), while neurotic, anxiety and somatoform disorders (F40-F48) predominated in the surgical group. The difference was not statistically significant $(P=0.06)$, except for when it was stratified for young 
Table I. Results.

Most frequent reasons for consultation (non-psychiatric staff)

Depression

Behavioral disorder due to confusion of agitation

$19.4 \%$

Treatment control/ psychiatric history

$16.3 \%$

Problems to reach a differential diagnosis

$14.4 \%$

Problems in the doctor-patient relationship, with information, refusal to carry out tests/treatments $\quad 10.6 \%$

Substance abuse / dependence

Diagnostic group ICD-10 of greatest prevalence (psychiatric diagnosis) [5]

Organic mental disorders (F00-F09)

$8.8 \%$

Adjustment disorders (F43.2)

Mood disorders (F10-F19)

$28.8 \%$

$22.0 \%$

$13.2 \%$

Substance abuse / dependence (F10-F19)

$12.5 \%$

Concordance between non-psychiatric and psychiatric staff

Substance abuse / dependence (F10-F19)

Mood disorders (F30-F39)

Neurotic and related disorders (F40-F48)

Organic mental disorders (F00-F09)

sensitivity $73.7 \%$

sensitivity $63.2 \%$

sensitivity $55.3 \%$

sensitivity $45.7 \%$ patients. This was mainly due to substance-related disorders where medical patients were concerned, and to anxiety and organic mental disorders with surgical patients. It has also been pointed out that surgery is related to different kinds of anxiety (before, during and postoperatory). The worry about not being self-sufficient and having to depend on others, the fear of death, pain, anesthesia, the loss of body parts or functions, postoperatory recovery, etc, all represent common reasons for anxiety states. Frequently, post-operatory confusional states are also common. This fact could also explain the gender differences amongst medical and surgical patients: substance abuse is more common in men and neurotic-anxiety disorders in women.

\section{REFERENCES}

1 Cirera-Costa E. Introducción. In Rojo-Rodes JE, Cirera-Costa E eds. Interconsulta psiquiátrica. Barcelona: Masson; 1997. p. 3-20.

2 Clarke DM, Smith GC. Consultation-liaison psychiatry in general medical units. Aust N ZJ Psychiatry 1995; 29 : 424-32.

3 Lipowski ZJ. Consultation-liaison psychiatry: the first half century. Gen Hosp Psychiatry 1986; 8 : 305-15.

4 Margolis RL, Non-psychiatrist house staff frequently misdiagnose psychiatric disorders in general hospital inpatients. Psychosomatics $1994 ; 35: 485-91$.

5 World Health Organization (WHO). Trastornos Mentales y del Comportamiento: criterios diagnósticos de investigación, 10a edición (ICD-10). Madrid: Meditor; 1992. 